\title{
Implementation of Vertical Handoff Algorithm between IEEE 802.11 WLAN \& CDMA Cellular Network
}

\author{
Vidya s. Pande, Dr. N. N. Mhala \\ ${ }^{1}$ (Electronics Department, B. D. C. O. E / Nagpur university India) \\ ${ }^{2}$ (H. O. D. Electronics Department, B. D. C. O. E / Nagpur university India)
}

\begin{abstract}
Wireless communications is the fastest growing segment of the communications industry. Everyone wants the quality of services anytime \& anywhere. Wireless networks can integrate various heterogeneous radio access technologies as GSM, WLAN, Wimax etc. WiMAX is an IP based, wireless broadband access technology that provides performance similar to 802.11/Wi-Fi networks with the coverage and QOS (quality of service) of cellular networks. WiMAX is also an acronym meaning "Worldwide Interoperability for Microwave Access (WiMAX). The main promise of interconnecting these heterogeneous networks is to provide high performance in achieving a high data rate and support real time applications. These services require various networks (such as CDMA2000 and Wireless LAN) to be integrated into IP-based networks, which further require a seamless vertical handoff to $4^{\text {th }}$ generation wireless networks. When a mobile host $(\mathrm{MH})$ changes its point of attachment, its IP address gets changed. MH should be able to maintain all the existing connections using the new IP address. This process of changing a connection from one IP address to another one in IP network is called handoff. Vertical handoff is switching from one network to another while maintaining the session. Vertical Handoff (VHO) is a major concern for different heterogeneous networks. VHO can be user requested or based on some criteria already designed by the researcher of that particular algorithm. The main objective is to implement efficient \& effective handoff scheme between two heterogeneous network ie. 802.11 WLAN \& CDMA.
\end{abstract}

Keywords - CDMA , 4G, Vertical Handoff, WLAN

\section{INTRODUCTION}

Today's network is becoming more and more a combination of different wireless networks to prove wider coverage and higher bandwidth to the users. Thus this heterogeneous property of network is giving wireless overlay network [1]. When a network is combination of multiple subnetworks and their coverage areas are overlapping, that combination of network is called overlay network. Usually heterogeneous networks are overlay network and most of the vertical handoff schemes are based on the overlay network format.

Various wireless LAN services are being introduced in hotspot areas, such as campuses, hotels and offices. In particular, IEEE802.11 WLAN services having high bandwidth are used to cover limited hotspot areas. If the mobile host $(\mathrm{MH})$ goes out of the hotspot coverage, the call will be dropped. In the 4th generation, a WLAN cell is overlaid within a CDMA2000 cell that is constructed into an ALL-IP based network. A seamless handoff is supplied through the vertical handoff process even if the MH goes out of the WLAN coverage area. The minimized cell size in 4th generation networks results in frequent handoffs. 4G Networks are all IP based heterogeneous networks that allow users to use any system at anytime and anywhere. Users carrying any integrated terminal can use a wide range of applications provided by multiple wireless networks. $4 \mathrm{G}$ systems provide not only telecommunications services, but also a data-rate service when good system reliability is provided. $4 \mathrm{G}$ networks face number of challenges in providing service anywhere and anytime. An event when a mobile station moves from one wireless cell to another is called Handoff. Handoff can be of two types first one is horizontal (intra-system) and second is vertical (inter-system) cases. Handoff within the same wireless access network technology is considered as horizontal handoff, and handoff among heterogeneous wireless access network technologies is considered vertical handoff. The development of WLAN began with the implementation of first IEEE 802.11 WLAN standards. The 802.11 standard have the utmost throughput up to 1 to $2 \mathrm{Mbits}$ per second and operate in $2.4 \mathrm{GHz}$ frequency. The 802.11 standard is based on radio technologies. WLAN intention was just to provide the wireless network infrastructure analogous to wired Ethernet network[2].

The fiber optic transport services providing the high bandwidth and data rates is replaced by WIMAX wireless technology all across the world. WIMAX is emerging technology to fulfill the high data rate and QoS requirements of the customers, also it is the cheap deployment of voice services with no need of line of sight wireless channel. WIMAX signals have the property to adopt the atmospheric conditions everywhere. WIMAX electromagnetic waves also offer the support of adoptive coding and different operation modes, so voice and data services can easily be transported by WIMAX network platform, [3]. 


\subsection{Handoff Methods}

Handoffs have several methods and they are technology dependent. The main handoff methods are as follows.

\subsubsection{Hard Handoff}

It has a brief disruption of service as it has to break before a making a switching action. Hard Handoffs are used by Time Division Multiple Access (TDMA) and Frequency Division Multiple Access(FDMA) systems.

\subsubsection{Soft Handoff}

It has no disruption of service action as it makes a switching action before the break. Multiple network resources are used by soft handoffs. Soft handoffs are used by CDMA system.

\subsubsection{Horizontal Handoff}

A horizontal handoff is a handoff between two network access points that use the same network technology and interface. For example, when a mobile device moves in and out of various $802.11 \mathrm{~b}$ network domains, the handoff activities would be considered as a horizontal handoff, since connection is disrupted solely by device mobility.

\subsubsection{Vertical Handoff}

A Vertical handoff is a handoff between two network access points, which are using different connection technologies. For example, when mobile device moves out an 802.11 network into a CDMA network, the handoff would be considered a vertical handoff.

In this paper, we have varied the parameters like bandwidth, node interest, quality of interest, received signal strength indication, \& network coverage.

\section{VERTICAL HANDOFF STEPS}

The vertical handoff [4] process involves three stages.

\subsection{Node formation \& Network discovery.}

In this phase, Mobile Nodes (MN) periodically searches if there are some other different types of wireless networks and take these discovered networks as candidates.

\subsection{Handoff decision}

In this phase where MNs compare the state of the current network with candidates, and select one as the handoff target from them according to a certain criterion.

\subsection{Handoff implementation} network.

In this phase where MNs execute the handoff actions and associate with the newly authenticated

The two cases in which handoffs occur. First, The process by which a MH that is in the WLAN service area leaves out from the area and connects to the CDMA Cellular network is called MD handoff. Second is When the MH serving in the CDMA cellular network region enters the WLAN service region, it connects to the WLAN. This is called the MU handoff.

\section{HANDOFF PARAMETERS}

We use set of parameters that can be used to make the vertical handoff.

\subsection{Bandwidth}

Different networks have different bandwidth, in an overlay network. Network bandwidth refers to the volume of data being transmitted across a network at any given point in time. When a MH moves into the coverage of a network with a higher bandwidth, it may connect to the higher bandwidth network via a downward handoff. When the MH moves out of its coverage, it can again connect to the original connection via an upward handoff.

\subsection{Received signal strength indication}

In an IEEE 802.11 system RSSI is the relative received signal strength in a wireless environment, in arbitrary units. RSSI is an indication of the power level being received by the antenna. Therefore, the higher the RSSI number, the stronger the signal. RSSI can be used internally in a wireless networking card to determine when the amount of radio energy in the channel is below a certain threshold at which point the network card is clear to send (CTS). Once the card is clear to send, a packet of information can be sent. The end-user will likely observe a RSSI value when measuring the signal strength of a wireless network through the use of a wireless network. If an MH finds the strength of the received signal from a network is below a threshold or below the strength of signal from another network, the MH may decide to change the network. 


\subsection{Quality of service ( qos)}

Vertical handoff decision can be based on the service an MH is using. Every service requires a certain level of QoS. If MH is using a more demanding service, like internet telephony, it may do a downward handoff the high bandwidth service. On the other hand, if the MH is using a service like web browsing, it may not decide to handoff if it meets the required QoS and avoid the necessary complications required for handoff.

\subsection{Network interest \& network coverage}

Network conditions, like congestion and traffic load may be deciding factor of a vertical handoff. Even if a MH can connect to a higher bandwidth network, but the network may be saturated and have high packet loss. In that case, the MH may decide against a handoff. Network coverage \& network interest should be proper.

\section{Features of $4 G$ Networks}

\section{LITERATURE SURVEY}

\subsection{High Speed}

$4 \mathrm{G}$ systems should offer a peak speed of more than 100Mbits per second in stationary mode with an average of $20 \mathrm{Mbits}$ per second when travelling.

\subsection{High Network Capacity}

It Should be at least 10 times that of $3 \mathrm{G}$ systems. This will quicken the download time of a 10-Mbyte file to one second on $4 \mathrm{G}$, from 200 seconds on $3 \mathrm{G}$, enabling high-definition video to stream to phones and create a virtual reality experience on high-resolution handset screens.

\subsection{Fast/Seamless handover across multiple networks}

$4 \mathrm{G}$ wireless networks should support global roaming across multiple wireless and mobile networks.

\subsection{Next-generation multimedia support}

The underlying network for $4 \mathrm{G}$ must be able to support fast speed volume data transmission at a lower cost than today.

The goal of $4 \mathrm{G}$ is to replace the current proliferation of core mobile networks with a single worldwide core network standard, based on IP for control, video, packet data, and voice. This will provide uniform video, voice, and data services to the mobile host, based entirely in IP. The objective is to offer seamless multimedia services to users accessing an all IP based infrastructure through heterogeneous access technologies. IP is assumed to act as an adhesive for providing global connectivity and mobility among networks. An all IP-based $4 \mathrm{G}$ wireless network has inherent advantages over its predecessors. It is compatible with, and independent of the underlying radio access technology [5].

In first paper, author presented a seamless vertical handoff procedure and the effective handoff algorithm for the handoff transition region between the WLAN and CDMA cellular network. Mobility management using MA and SA was also adopted to minimize the handoff delay in the WLAN-CDMA Cellular interconnection architecture based on IP. In the handoff algorithm they use the number of continuous beacon signals whose signal strength from the WLAN falls below the predefined threshold value.

In second paper proposed a handoff decision process with network selection that decides the optimal velocity threshold in order to improve GoS and minimize the number of handoff attempts with a given traffic volume in a WLAN \& CDMA integrated network. The simulation results show the dependency of the system performance upon the velocity threshold $V T$. The velocity threshold has been shown to be an important system parameter that the system provider should determine to produce better $\mathrm{GoS}$ and lower handoff rates. From the simulation results, they were able to validate the procedures determining the optimal $V T$, which depends upon $\mathrm{GoS}$ as well as the number of handoff attempts.

Third paper outlines a handover strategy for heterogeneous wireless networks. The algorithms presented above provide capacity where it is needed and when it is needed. Moreover more clients are satisfied and the overall utilization of the network is improved. Cell breathing is implemented by adjusting the power of each AP / BS in the network. The dynamic version of the algorithm can adapt to changes in client demands by maximizing the total satisfied demand while limiting the number of clients that switch APs or BSs.

In fourth paper next generation wireless systems consist of heterogeneous wireless networks. Wireless LAN (WLAN) and cellular mobile radio systems will be important part of heterogeneous networks. Universal mobile telecommunication system (UMTS) provides wide-area coverage, high mobility but relatively low data rate. WLAN is designed for low-range, high/medium data rate access, low mobility and can be used as a complement to larger cellular mobile radio systems. In this paper, they consider a simple heterogeneous system of WLAN and UMTS cell. Vertical handover plays a vital role in ensuring quality of service in heterogeneous networks. In this paper, we proposed a vertical handover algorithm for an integrated cellular wireless LAN 
system. The design goal is to provide the user the best available quality of service (QoS) at any time. Moreover, connections are handed over independently between UMTS and WLAN, aiming at seamless service continuity. Here they have taken handover rate, probability of outage and probability of handover as the performance metrics. Simulation results show that adaptive vertical handover algorithm improves the performance.

In last paper, wireless communications is the fastest growing segment of the communications industry. As such, it has captured the attention of the media and the imagination of the public. Cellular systems have experienced exponential growth over the last decade and there are currently around two billion users worldwide. In this paper they use the vertical handoff algorithm and make a network based on WLAN and CDMA network and then analyse on the basis of this network by using the formula throughput.

\section{VERTICAL HANDOFF ALGORITHM}

The automatic 'fall over' from one technology to another in order to maintain communication, Vertical handovers is used [7]. Vertical handoff refers to a network node changing the type of connectivity it uses to access a supporting infrastructure, usually to support node mobility. A Vertical handoff is a handoff between two network access points, which are using different connection technologies. For example, when mobile device moves out an 802.11 network into a GPRS network, the handoff is called a vertical handoff. We have considered two network first one is CDMA network \& second is WLAN 802.11. Vertical handoff algorithm is explained by considering some conditions as follows. First we considered, currently nodes are present in CDMA network .

If node bandwidth is less than 15 ORing with node interest is less than 0.5 ORing with node quality of service is less than 0.5 ORing with node received signal strength indication is less than -70 ORing with node network coverage is less than 150 .

Here We have considered various parameters \& compared with some threshold values. If this above condition is satisfied then node will change network i.e. from CDMA to WLAN 802.11 \& vice versa.

\section{RESULTS}

In the simulation of vertical handoff algorithm, parameters of random nodes are varying. We use two networks first one is CDMA and Second is WLAN. We use some threshold value of various parameters. By comparing these parameters, the node will choose current network.

For creating nodes first we have to create user datagram Protocol agent i.e. UDP after creating UDP, it attach to node. Then create constant bit rate ratio i.e. CBR traffic source. It attach to UDP. Connect traffic source to traffic sink. At last create link between nodes. We Use Ad hoc on demand distance vector protocol i..e AODV. The Nam file of node formation in which network animation of various nodes are shown.

\subsection{Implementation of vertical handoff algorithm}

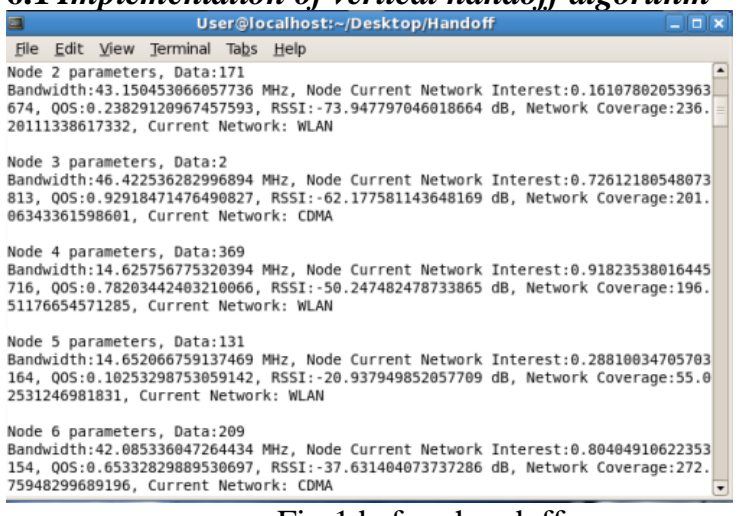

Fig.1 before handoff

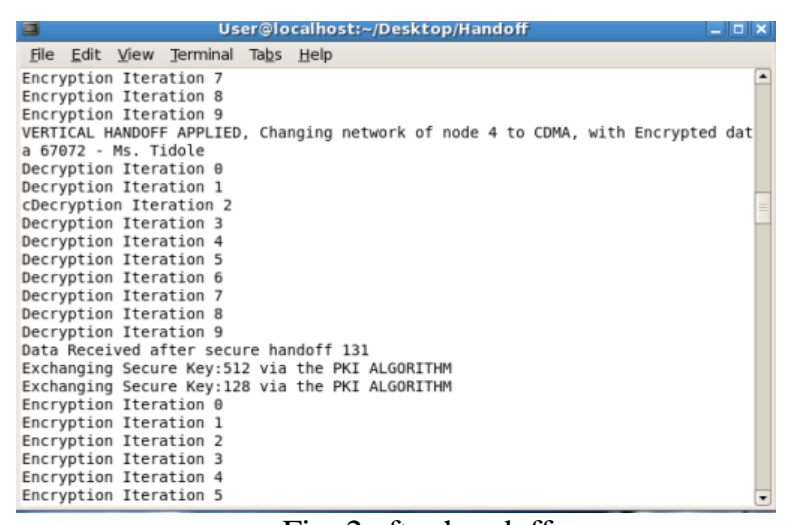

Fig. 2 after handoff

Above fig. 1 shows nodes changes parameters before applying vertical handoff. In this fig. 1 we consider node 4 Parameters. As per changing parameters bandwidth is 14.62 in $\mathrm{MHz}$, node current network interest is 0.91 , quality of service is 0.78 , received signal strength indication is -50.24 in $\mathrm{dB}$, network coverage is 196.51 \& current network is WLAN.

Before applying vertical handoff algorithm, in fig .1, node 4 is in WLAN network . After applying vertical handoff algorithm, In fig .2, node 4 changed network from WLAN to CDMA with encrypted data 67072. 


\section{GRAPHS}

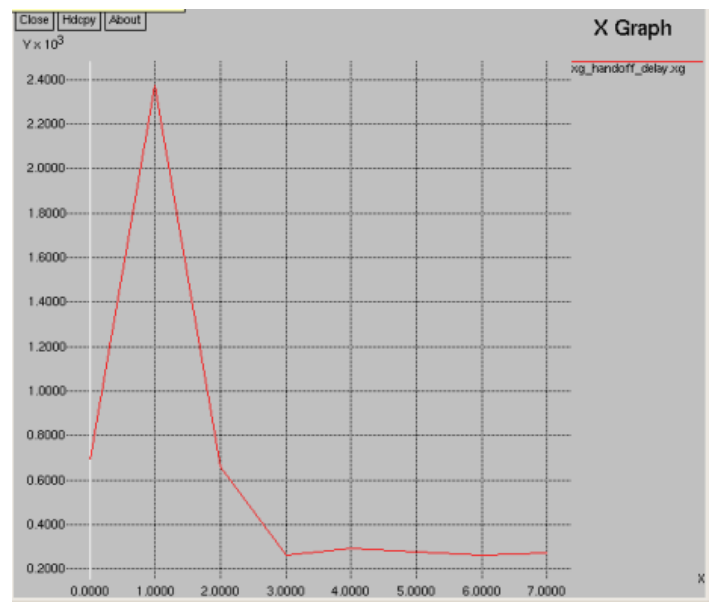

Fig. 3 Handoff delay vs .time

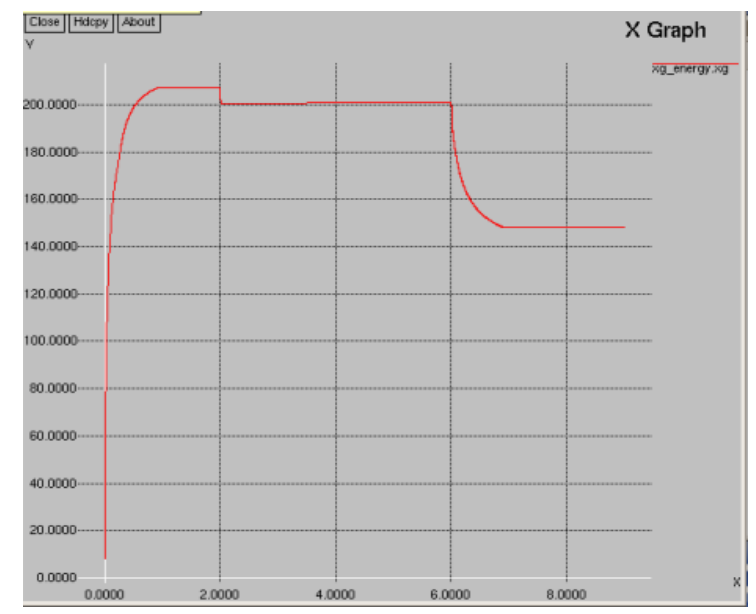

Fig. 4 Energy vs time

In fig. 3, graph is of handoff delay vs time .In this graph time is considered on X-axis \& handoff delay is considered on Y-axis. As the time increases handoff delay also increases up to the threshold value but after that handoff delay decreases.

In fig. 4 , the is a graph between energy vs time. On X-axis time is taken \& on Y -axis energy is taken which is in joules. As time increases, energy starts increases initially after some time it becomes constant.

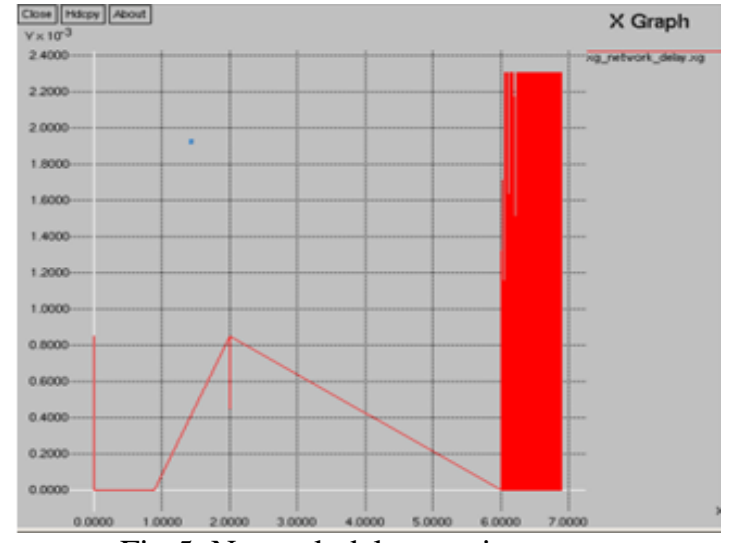

Fig.5 Network delay vs. time

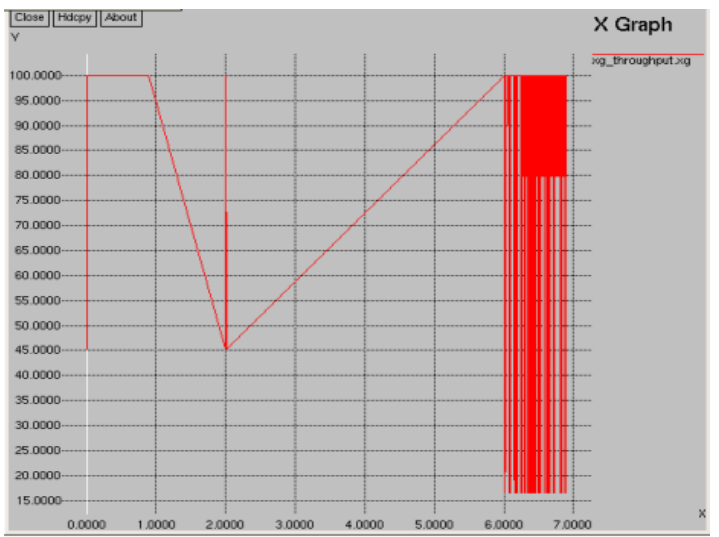

Fig . 6 Throughput vs. time

In fig 5, the graph is between network delay vs time. On $\mathrm{X}$ - axis time has taken \& on $\mathrm{Y}$ - axis network delay has taken. As the time increases, initially network delay becomes constant after some time it starts to increases.

In fig.6 the graph is between throughput \& time. Throughput is on Y-axis \& time is taken on X-axis. Initially the time increases, throughput is constant after some time it slows down. Throughput is the amount of data transferred from one place to another or processed in a specified amount of time. Data transfer rates for disk drives and networks are measured in terms of throughput. Typically, throughputs are measured in kbps, Mbps and Gbps. 


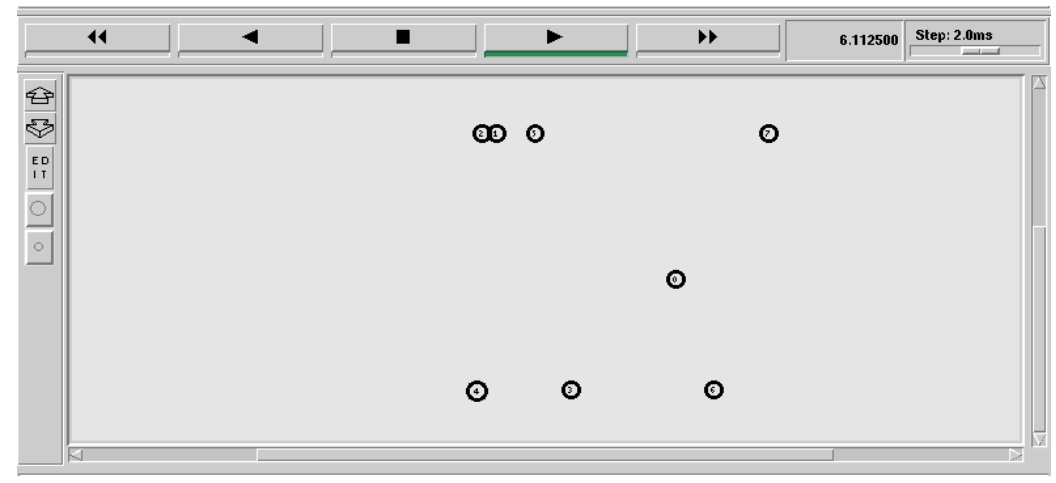

Fig.7 Vertical handoff animation

This is animation file after applying vertical handoff algorithm. In this animation file, nodes changes its network when the condition satisfied. When play button starts, nodes will move from one network to another.

\section{CONCLUSION}

In this paper, we presented a formation of random nodes by changing various parameters such as bandwidth, node current network interest, quality of services, received signal strength indication, \& network coverage. With the popularity of many types of networks, issues of vertical handoff are getting more and more important. In our module, we are comparing various parameters with some threshold values. If conditions are satisfied, then node will change current network to another network. For security perpose we can use encryption $\&$ decryption code. If the parameters are chosen correctly the algorithm can perform quite efficiently and a maximum performance can be extracted from the device given an operating environment.

One major drawback is that if the parameters are not chosen rightly the algorithm would result in an inefficient usage of resources and a sub-optimal performance of the device into which the algorithm is loaded.

Handoff delay poses an important QoS-related issue in $4 \mathrm{G}$ wireless networks. During the handoff process, the user may experience a significant drop in QoS that will affect the performance of both upper-layer protocols and applications. Deploying a priority-based algorithm and using location aware adaptive applications can reduce both handoff delay and QoS variability. When there is a potential for considerable variation between senders' and receivers' device capabilities, deploying a receiver-specific filter in part of the network close to the source can effectively reduce the amount of traffic and processing, satisfying other users' QoS needs. Although 4G wireless technology offers higher bit rates and the ability to roam across multiple heterogeneous wireless networks, several issues require further research and development. The design \& working of vertical handoff algorithm will be under NS-2 platform in the LINUX environment.

\section{REFERENCES}

[1]. M. Stemm and R. Katz, "Vertical handoffs in wireless overlay networks," ACM Mobile Networking(MONET) Special Issue on Mobile Networking in the Internet, vol. 3, no. 4, no. 4, p. 335350,1998.

[2]. Bradley Mitchell; "Wireless Standards 802.11b, 802.11a, 802.11g and 802.11n"

[3]. Dayou Quan, Cavendish, Ting Wang, "WIMAX Services over Transport Networks", NEC, USA.

[4]. Sun Wireless, "All IP Wireless, All the Time - Building a 4th generation wireless network with open systems solutions". Availableat :http://research.sun.com/features/4g_wireless/

[5]. Mandeep Kumar Gondara,"Requirements of Vertical Handoff Mechanism in 4G Wireless Networks" in International Journal of Wireless \& Mobile Networks, April 2011

[6]. Hyosoon Park, Sunghoon Yoon, Taehyoun Kim , Jungshin Park, Misun Do, and Jaiyong Lee "Vertical Handoff Procedure And Algorithm Between IEEE 802.11 WLAN And CDMA Cellular Network".2003

[7]. Richa Agarwal and Inderjeet Kaur "Comparative Analysis Of Vertical Handoff In IEEE 802.11 WLAN And CDMA Network" August 2012

[8]. N. P. Singh and Brahmjit Singh "Improved Vertical Handover Decision Algorithm for UMTS-WLAN" December, 2011

[9]. Jang-Sub Kim, Erchin Serpedin "Handoff Triggering and Network Selection Algorithms for Load-Balancing Handoff in CDMAWLAN Integrated Networks" 11 August 2008

[10]. R. Berezdivin, R. Breinig, and R. Topp, "Next- Generation Wireless Communications Concepts and Technologies," IEEE Communications Magazine, March 2002

[11]. T. Otsu, I, Okajima, N. Umeda, and Y. Yamao, "Network Architecture for Mobile Communications Systems Beyond IMT-2000," IEEE Personal Communications, October 2001

[12]. Mi-Sun Do, Youngjun Park, and Jai-Yong Lee," Channel Assignment with QoS Guarantees for a Multi-Class Multi-Code CDMA System," accepted for publication in IEEE Transactions on Vehicular Technology. 